\title{
Retinal Nerve Fibre Layer Analysis in Non- Glaucomatous Pseudoexfoliation Syndrome Patients
}

\author{
Erum Waris Khateeb ${ }^{1}$ Imtiyaz Ahmad Lone ${ }^{1} \quad$ Ifrah Ahmad Qazi ${ }^{2}$ \\ ${ }^{1}$ Department of Ophthalmology, Sher-e-Kashmir Institute of Medical \\ Sciences Medical College and Hospital, Srinagar, Jammu and \\ Kashmir, India \\ 2 Department of Urology, Narayana Medical College, Nellore, Andhra \\ Pradesh, India

\begin{abstract}
Address for correspondence Imtiyaz Ahmad Lone, MS, Department of Ophthalmology, Sher-e-Kashmir Institute of Medical Sciences Medical College and Hospital, Srinagar, Jammu and Kashmir 190011 India (e-mail: imtiyazalone@yahoo.co.in).
\end{abstract}

Int J Recent Surg Med Sci 2022;8:66-69.

\begin{abstract}
Background Pseudoexfoliation syndrome (PXS) is a disorder characterized by the progressive accumulation of fibrillary extracellular deposits in several ocular tissues. It is an independent risk factor for glaucomatous optic nerve damage. Retinal nerve fiber layer (RNFL) thickness analysis using optical coherence tomography is a documented investigative tool to detect glaucoma at an early stage.

Objective The aim was to evaluate and compare RNFL thickness in PXS patients without glaucoma with their age- and sex-matched healthy controls and detect the possibility of early glaucomatous damage in patients with RNFL thinning.

Study Design This was a cross sectional case-control study.

Materials and Methods A total of 100 patients were included, of which 50 were cases (Group A) and 50 were controls (Group B). RNFL thickness of cases and controls were compared using Zeiss Cirrus HD-OCT 500 (ZEISS Medical Technology, United States). Results There were no significant differences between the two groups with respect to mean RFNL thickness in nasal $(p=0.129)$ and temporal quadrants $(p=0.832)$. The

Keywords

- pseudoexfoliation syndrome

- glaucoma

- retinal nerve fiber layer mean inferior RNFL thickness values were $112.9 \pm 21.72 \mu \mathrm{m}$ in Group $A$ and $120.6 \pm 10.35 \mu \mathrm{m}$ in Group B $(p=0.002)$. The mean thickness of the retinal nerve fiber layer in superior quadrant in Group $A$ was $101.6 \pm 23.16 \mu \mathrm{m}$, whereas it was $113.5 \pm 13.47 \mu \mathrm{m}$ in group $B(p<0.001)$. The average RFNL thickness in Group A was $85.1 \pm 13.99 \mu \mathrm{m}$ and it was $88.9 \pm 7.01 \mu \mathrm{m}$ in Group B $(p=0.017)$.

Conclusion There was statistically significant difference in RNFL thickness between cases and controls in inferior and superior quadrants and in global average thickness.
\end{abstract}

\section{Introduction}

Glaucoma is a leading cause of irreversible blindness throughout the world. ${ }^{1}$ Pseudoexfoliation syndrome (PXS) is the most common identifiable cause of open-angle glaucoma worldwide. ${ }^{2}$ PXS is an age-related, genetically deter- mined, generalized disorder of the extracellular matrix characterized by production and progressive accumulation of a fibrillar material in ocular tissues, skin, and other visceral organs. Its ocular manifestations affect all the structures of the anterior segment as well as conjunctiva and
DOI https://doi.org/ $10.1055 / \mathrm{s}-0041-1739373$ ISSN 2455-7420.

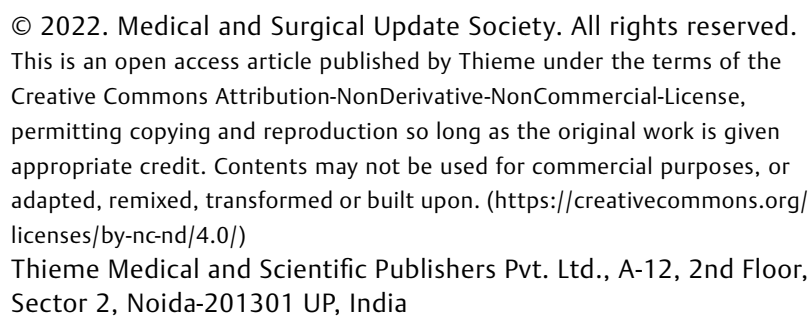


orbital tissues. ${ }^{3}$ It is the optic nerve head (ONH) and the retinal nerve fiber layer (RNFL) containing retinal ganglion cell axons that are most clearly associated with glaucomatous vision loss, especially the circumpapillary RNFL. ${ }^{4}$ Pseudoexfoliative glaucoma (PXG) tends to progress slowly and patients are often asymptomatic until the disease reaches an advanced stage. PXG represents a relatively severe and progressive type of glaucoma, with a generally poor prognosis due to high intraocular pressure levels, great pressure differences between the two eyes, and fluctuations in the diurnal pressure curve..$^{5}$ Optic neuropathy due to glaucoma is largely irreversible, so early detection and prevention is of vital importance.

Structural damage of ONH and RNFL may precede functional loss and it is estimated that between 30 and $50 \%$ of retinal ganglion cells may be lost before detectable changes in visual fields are evident. ${ }^{6-8}$ Spectral domain optical coherence tomography (SD-OCT) provides highresolution, quantitative, and reproducible measurements of RNFL having high sensitivity and specificity for differentiating normal eyes from patients with early glaucoma. OCT has a well-known role in diagnosing pre-perimetric glaucoma. ${ }^{6}$

\section{Aim}

This study was done to compare the mean RNFL thickness in patients having PXS with normal age-matched healthy controls using SD-OCT.

\section{Materials and Methods}

This case-control study was conducted at a tertiary care center in the Postgraduate Department of Ophthalmology from October 2017 to April 2019, after approval from hospital's ethics committee. A written informed consent was obtained from the patients. A total of 100 patients were included in this study, of which 50 were cases (Group A), which included nonglucomatous PXS patients and 50 were their age- and sex-matched healthy controls (Group B). Eighty-eight eyes in Group A were examined, as 12 patients had unilateral pseudoexfoliation and 100 eyes in Group B were examined. The diagnosis of pseudoexfoliation (PEX) was made on the basis of following findings: PEX material on the lens capsule or near the pupil; transillumination defects near the pupil; increased pigmentation or PEX material at the angle, or both. An eye was considered normal if it had an IOP of $<21 \mathrm{~mm} \mathrm{Hg}$, an optic disc with normal ophthalmoscopic appearance, and normal visual field test results. All participants underwent a complete ocular examination. Biomicroscopic and fundoscopic examination with a 90 dioptre lens was performed, and IOP was measured using the Goldmann applanation tonometer. Visual field evaluation was done using the 30-2 SITA-Standard algorithm (Humphrey Visual Field Analyzer). OCT was done by Cirrus HD 500 spectral domain OCT (ZEISS Medical Technology, United States).

\section{Inclusion Criteria}

- Presence of pseudoexfoliative material on the pupil margin or on the lens capsule on bio-microscopic evaluation.

- Intraocular pressure equal to or less than $21 \mathrm{~mm} \mathrm{Hg}$.

- Normal optic nerve head appearance (with C/D ratio 0.3-0.4).

- Normal visual field analysis on Humphrey field analyzer.

\section{Exclusion Criteria}

- Glaucomatous patients or family history of glaucoma.

- Media opacity interfering with visualization and OCT image capturing.

- History of any ocular diseases.

- Previous ocular trauma.

- Any retinal pathology.

- History of diabetes mellitus.

All data were analyzed using the SPSS software 20.0 (SPSS Inc., Chicago, Illinois, USA). Continuous variables are expressed as mean \pm standard deviation (SD) and categorical variables are summarized as frequencies and percentages. Student's independent $t$-test was employed for comparing continuous variables. Chi-square test or Fisher's exact test, whichever appropriate, was applied for comparing categorical variables. Fundus changes were compared using MannWhitney $U$ test. A $p$-value of less than 0.05 was considered statistically significant. All $p$-values were two tailed.

\section{Results}

Mean age of Group A (cases) was $63.3 \pm 6.98$ years and of Group B (control) was $63.9 \pm 6.81$ years $(p=0.68)$, and most of the patients were in the range of 60 to 69 years. Both groups were gender matched with a female preponderance. The mean IOP was comparable in both groups. The mean IOP in the PEX group was $16.91 \pm 1.76 \mathrm{~mm} \mathrm{Hg}$ and $16.85 \pm 1.91 \mathrm{~mm} \mathrm{Hg}$ in the control group $(p=0.83)$ ( - Table 1). The mean RNFL measurements at each location are shown in - Table 2 . The mean superior RNFL thickness was $101.6 \pm 23.16 \mu \mathrm{m}$ in Group A and $113.35 \pm 13.47 \mu \mathrm{m}$ in Group B $(p<0.001)$. The mean inferior RNFL thickness was $112.9 \pm 21.72 \mu \mathrm{m}$ in Group A and $120.6 \pm 10.35 \mu \mathrm{m}$ in Group $\mathrm{B}(p=0.002)$. The mean nasal RNFL thickness was $63.1 \pm 10.9$ $\mu \mathrm{m}$ in Group A and $63.4 \pm 9.98 \mu \mathrm{m}(p=0.129)$ in Group B. The mean temporal RNFL thickness was $60.7 \pm 20.33 \mu \mathrm{m}$ and

Table 1 Patient demographic profile with intraocular pressure

\begin{tabular}{|c|c|c|c|c|}
\hline & \multicolumn{2}{|c|}{ Cases (Group A) } & \multicolumn{2}{|c|}{$\begin{array}{l}\text { Controls } \\
\text { (Group B) }\end{array}$} \\
\hline $\begin{array}{l}\text { Age in years } \\
(\text { mean } \pm \text { SD) }\end{array}$ & \multicolumn{2}{|c|}{$63.3 \pm 6.98$} & \multicolumn{2}{|c|}{$63.9 \pm 6.18$} \\
\hline \multirow[t]{2}{*}{ Gender } & $48 \%$ & Males & $46 \%$ & Males \\
\hline & $52 \%$ & Females & $54 \%$ & Females \\
\hline $\begin{array}{l}\text { IOP in } \mathrm{mm} \mathrm{Hg} \\
(\text { mean } \pm S D)\end{array}$ & \multicolumn{2}{|c|}{$16.91 \pm 1.759$} & \multicolumn{2}{|c|}{$16.85 \pm 1.909$} \\
\hline
\end{tabular}


Table 2 Quadrant-wise RNFL thickness

\begin{tabular}{|c|c|c|c|}
\hline RNFL thickness & $\begin{array}{l}\text { Cases (Group A) } \\
N=88 \text { (eyes) }\end{array}$ & $\begin{array}{l}\text { Controls (Group B) } \\
N=100 \text { (eyes) }\end{array}$ & $p$-Value \\
\hline Superior $(\mu \mathrm{m})$ & $101.6 \pm 23.16$ & $113.5 \pm 13.47$ & $<0.001$ \\
\hline Inferior $(\mu \mathrm{m})$ & $112.9 \pm 21.72$ & $120.6 \pm 10.35$ & 0.002 \\
\hline Nasa $(\mu \mathrm{m})$ & $63.1 \pm 10.9$ & $63.4 \pm 9.98$ & 0.129 \\
\hline Temporal $(\mu \mathrm{m})$ & $57.5 \pm 13.12$ & $57.3 \pm 9.56$ & 0.925 \\
\hline Global average $(\mu \mathrm{m})$ & $85.1 \pm 13.99$ & $88.9 \pm 7.01$ & 0.017 \\
\hline
\end{tabular}

$57.3 \pm 9.56 \mu \mathrm{m}$ in Group A and B, respectively $(p=0.832)$. The mean average peripapillary RNFL thickness was $85.1 \pm 13.99$ $\mu \mathrm{m}$ in Group A and 88.9 $\pm 7.01 \mu \mathrm{m}$ in Group B $(p<0.10)$ (-Table 2; -Fig. 1).

\section{Discussion}

Secondary chronic open-angle glaucoma associated with PXS accounts for $\sim 25 \%$ of all glaucoma and represents the most common identifiable cause of glaucoma overall. ${ }^{9}$ Optic disc evaluation by slit lamp biomicroscopy or photography is subjective and causes inter-observer variability. Gold standard test for detection of glaucomatous optic nerve damage is visual field analysis; however, abnormality is detected only after $40 \%$ loss of retinal ganglion cell has occurred. Glaucoma is identified biologically by the death of retinal ganglion cells. As retinal ganglion cells are lost, the retinal nerve fibers are also lost, and this layer thins. Thus, RFNL defect is a main sign of early glaucomatous damage and that visual field testing, fundus image, and IOP may not be sensitive to detect this early damage. Structural damage of ONH and RNFL may precede functional loss. ${ }^{6-8}$ The high diagnostic accuracy of the SD OCT allows for rapid, reproducible OCT scanning of the
RNFL thickness and monitors changes in thickness for the detection of early glaucoma. ${ }^{10,11}$

The aim of this study was to detect early RNFL damage in pseudoexfoliative patients without glaucoma by measuring the RNFL thickness using SD OCT and comparing the results with age-matched healthy control subjects. In our study, the mean RFNL thickness in the inferior quadrant showed statistically significant thinning in patients with PXS versus healthy controls (112.9 vs. $120.6 ; p=0.002$ ). The present study also showed statistically significant thinning of RNFL in superior quadrant of BOTH PXS and control groups (101.6 vs. 113.5; $p<0.001)$. The RFNL in the nasal quadrant was thinner in PXS group than in controls but was not statistically significant $(p=0.129)$. In temporal quadrant the RNFL was thicker in the PXS group but there was no statistically significant difference in the thickness of RNFL among PXS and controls groups (0.832). This study also revealed a thinner mean global average peripapillary RNFL thickness in the PXS group as compared with the control group (85.1 vs. 88.9)

In the early stage of PXG, RNFL defect usually progresses to affect mainly local areas in the superior and/or inferior pole. However, with more progression of disease, it becomes more extensive and shows diffuse and a combination of RNFL defects.

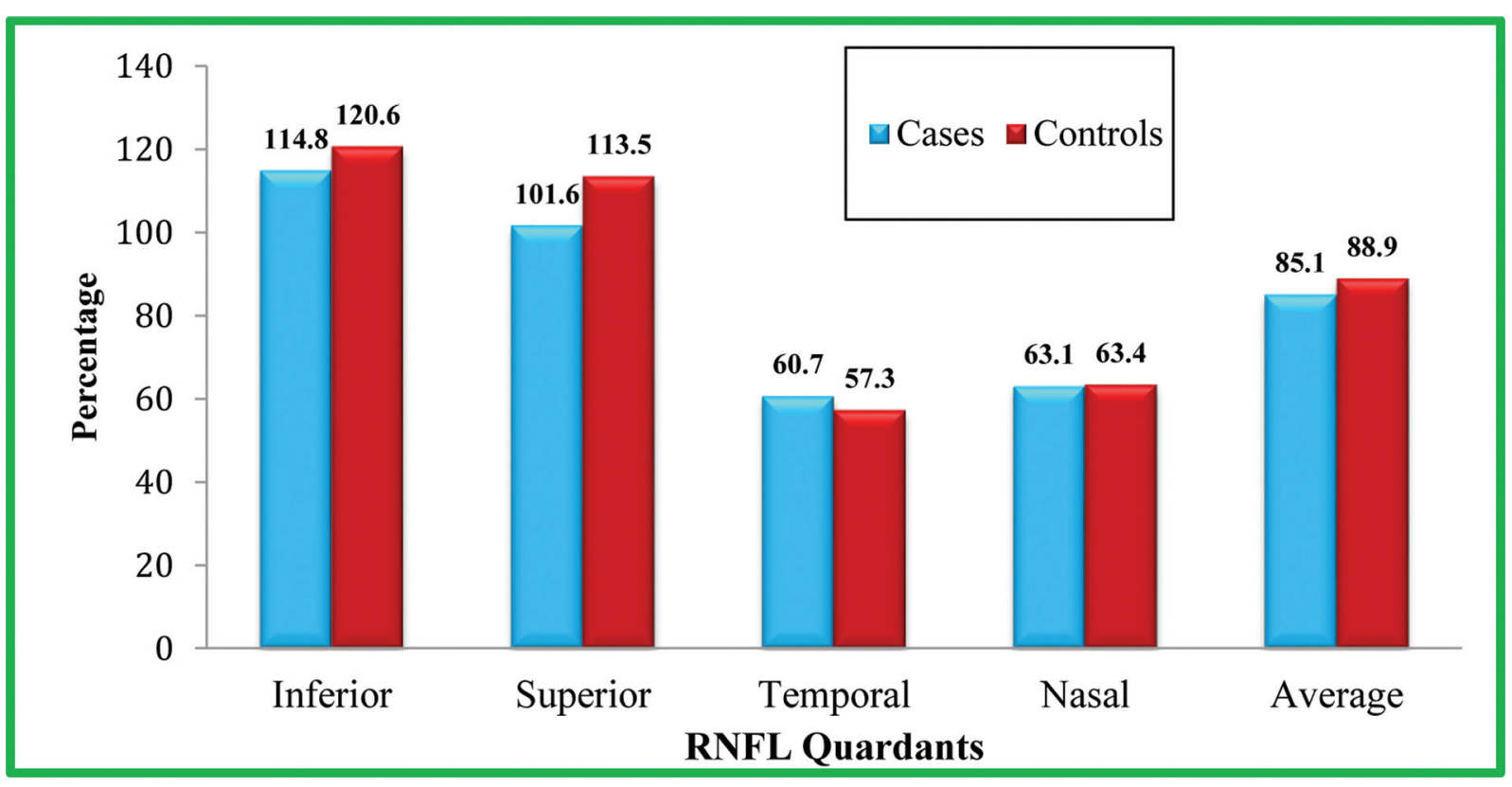

Fig. 1 Quadrant-wise comparison of RFNL thickness. 
This study revealed a thinner mean global average peripapillary RNFL thickness in cases as compared with the control group.

Naik et al $^{12}$ analyzed retinal nerve fiber layer thickness in patients with pseudoexfoliation syndrome using SD OCT. The RNFL thickness in normal subjects (group B); was normally thicker in the superior and inferior, thinner in the temporal and the lowest in the nasal quadrants; however in the PXS group, there were significant differences in the RNFL thickness among the four quadrants except for the nasal quadrant compared with the control group. This could be explained by the fact that higher axonal density and higher proportion of large fibers occupy the superior and inferior portions of the optic nerve head compared with the temporal and nasal portion. These fibers (superior and inferior) are, in addition, most susceptible to early glaucomatous damage.

In the study by Mohamed, ${ }^{13}$ the early glaucomatous changes in pseudoexfoliation syndrome patients was assessed. He found statistically significant thinning of RNFL in the temporal, superior, and inferior quadrants.

Another study was done by Nawaz et al $^{14}$ comparing the RNFL thickness in PXS patients with normal subjects. Although they found thinner mean RFNL thickness in all quadrants but the difference was statistically significant only in the inferior quadrant.

Most of the work done on measurement of RNFL thickness in PXS without glaucoma using different OCT machines revealed a thinner mean global peripapillary RNFL in the eyes with PXS as compared with the control group with some variations in quadrantic measurements that may be attributed to variation in age, sample size, ethnicity, and type of OCT machine used.

Although results of our study correspond with those of international studies, some variation in absolute values was found that may be attributed to variation in age, ethnicity, gender distribution, machine used, and normative data. This study gives some insight into diagnosing pre-perimetric glaucoma using OCT as PXS patients with normal IOP and visual fields were tested and found to be having a thinner RNFL than their age-matched adults.

\section{Conclusion}

The results of this study show that the eyes with PXS are associated with a significant decrease in RNFL thickness, and detecting this thinning in the eyes of PXS patients without glaucomatous change will help in early detection of patients at risk of glaucoma. Such PXS patients without glaucoma having RNFL thinning will be considered as a high-risk group for the development of glaucoma and will be kept on regular follow-up.

\section{Authors' Contributions}

E.W.K.: Materials, data collection, Writing; I.A.L.: conception, design, supervision, critical review; I.A.Q.: analysis and interpretation, literature review.

Conflict of Interest

None declared.

\section{References}

1 Thylefors B, Négrel AD, Pararajasegaram R, Dadzie KY. Global data on blindness. Bull World Health Organ 1995;73(01):115-121

2 Ritch R. Exfoliation syndrome-the most common identifiable cause of open-angle glaucoma. J Glaucoma 1994;3(02): 176-177

3 Ritch R, Schlötzer-Schrehardt U, Konstas AG. Why is glaucoma associated with exfoliation syndrome? Prog Retin Eye Res 2003; 22(03):253-275

4 Allingham R. Rand. Shields Textbook of Glaucoma. 6th ed. Philadelphia: Lippincott Williams \& Wilkins; 2011 Chapter 4: Optic nerve, Retina and Choroid; p51

5 Schlötzer-Schrehardt U, Küchle M, Jünemann A, Naumann GOH. [Relevance of the pseudoexfoliation syndrome for the glaucomas]. Ophthalmologe 2002;99(09):683-690

6 Gondal TM, Qazi ZU, Jamil AZ, Jamil MH. Accuracy of the retinal nerve fiber layer measurements by Stratus optical coherence tomography for perimetric glaucoma. J Coll Physicians Surg Pak 2011;21(12):749-752

7 Pan Y, Varma R. Natural history of glaucoma. Indian J Ophthalmol 2011;59(Suppl):S19-S23

8 Mansoori T, Viswanath K, Balakrishna N. Ability of spectral domain optical coherence tomography peripapillary retinal nerve fiber layer thickness measurements to identify early glaucoma. Indian J Ophthalmol 2011;59(06):455-459

9 Schlötzer-Schrehardt UM, Koca MR, Naumann GOH, Volkholz H. Pseudoexfoliation syndrome. Ocular manifestation of a systemic disorder? Arch Ophthalmol1992b;110(12):1752-1756

10 Streeten BW, Dark AJ. Pseudoexfoliation Syndrome. Chap. 19. In: Garner A, Klintworth GK, eds. Pathobiology of Ocular Disease. 2nd Ed. Part A. New York: Marcel Dekker Inc; 1994:591-629

11 Vizzeri G, Balasubramanian M, Bowd C, Weinreb RN, Medeiros FA, Zangwill LM. Spectral domain-optical coherence tomography to detect localized retinal nerve fiber layer defects in glaucomatous eyes. Opt Express 2009;17(05):4004-4018

12 Yüksel N, Altintaş O, Celik M, Ozkan B, Cağlar Y. Analysis of retinal nerve fiber layer thickness in patients with pseudoexfoliation syndrome using spectral domain optical coherence tomography. Int J Sci Res 2015;4(12):

13 Mohamed MM. Detection of early glaucomatous damage in pseudo exfoliation syndrome by assessment of retinal nerve fiber layer thickness. Middle East Afr J Ophthalmol 2009;16(03): 141-145

14 Nawaz S, Sofi RA, Querishi T. Evaluation of changes in retinal fiber layer thickness in pseudoexfoliation syndrome using spectral domain optical coherence tomography. Int J Med Sci Public Health 2018;7(07):529-532 